\title{
Derivada Topológica de Primeira e Segunda Ordem no Problema de Tomografia por Impedância Elétrica
}

\author{
Andrey Dione Ferreira, Antonio André Novotny \\ Coordenação de Matemática Aplicada e Computacional, LNCC \\ 25651-075, Petrópolis, RJ \\ E-mail: andreydf@lncc.br, novotny@lncc.br.
}

\begin{abstract}
Resumo: O problema de tomografia por impedância elétrica consiste em determinar a distribuição de condutividade elétrica de um meio a partir de medidas tomadas na fronteira do corpo. Esse problema tem diversas aplicações em medicina com a detecção de tumores e monitoramento de apneias, em geofísica e ciências ambientais com a localização de depósitos de minerais e monitoramento de fluídos, e na engenharia com a detecção de corrosões em estruturas. Neste trabalho, objetiva-se reconstruir um conjunto de inclusões com coeficiente de condutividade elétrica distinta do meio, submetendo o corpo a um conjunto de fluxo elétrico e medindo a correspondente distribuição de campo elétrico sobre sua fronteira. Como esse problema é escrito na forma de uma equação diferencial parcial sobredeterminada, a ideia básica consiste em reescrevê-lo na forma de um problema de otimização. Em particular, objetiva-se minimizar um funcional de forma que mede a diferença entre os potenciais medidos e calculados numericamente. Sobre a solução do problema inverso, ambos potenciais coincidem. Sendo assim, o referido funcional é minimizado utilizando o conceito de derivada topológica de primeira e segunda ordem. Cabe esclarecer que a derivada topológica de primeira ordem não fornece informação suficiente para resolução do problema inverso, apresentando seus valores críticos sobre a fronteira do domínio devido à elipticidade do problema.
\end{abstract}

Palavras-chave: Problemas Inversos, Tomografia por Impedância Elétrica, Análise de Sensibilidade Topológica

\section{Introdução}

O problema inverso de EIT (Electrical Impedance Tomography) consiste em determinar a condutividade elétrica de um corpo a partir de medições eletromagnéticas em sua superfície. Esse problema tem diversas aplicações, dentre as quais podemos citar as aplicações em ciências médicas com na detecção de tumores $[1,7]$ e monitoramento de apneias, em geofísica e ciências ambientais com a localização de depósitos de minerais e monitoramento de fluídos e na engenharia com a deteç̧ão de corrosões em estruturas.

Em comparação com outros métodos, tomografia por raio-X, por exemplo, é que a aplicação de correntes elétricas não causam efeitos colaterais, além de baixo custo em testes não destrutivos $[1,2,7]$.

\section{Formulação do Problema}

Descreve-se matematicamente o problema de EIT bem como a formulação do problema inverso envolvido. 


\subsection{Tomografia por Impedância Elétrica}

Considere um domínio $\Omega$ que representa um dado corpo que possua a capacidade de conduzir eletricidade em seu interior e cujo coeficiente de condutividade elétrica seja $k^{*}(x)$, com $x \in \Omega$. Assumindo que este corpo é submetido a um potencial elétrico conhecido $\varphi(\sigma) \operatorname{com} \sigma \in \partial \Omega$, onde $\partial \Omega$ representa a fronteira de $\Omega$, então o potencial elétrico $u(x)$ em $\Omega$ satisfaz a equação elíptica:

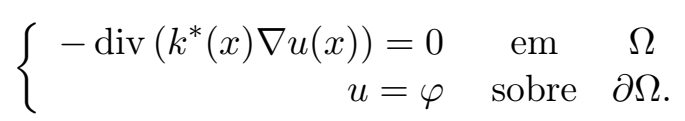

Nesse sentido, a intensidade (local, isto é em $\partial \Omega$ ) da corrente é representada por $k^{*} \partial_{n} u$, onde $\partial_{n} u$ denota a derivada normal exterior.

Assim, a questão que se coloca é a seguinte: a partir dessas informações, podemos determinar o coeficiente de condutividade $k^{*}(x)$ ? Essa questão nos leva naturalmente ao estudo do operador de Dirichlet-to-Neumann (DtN) $\Lambda_{k^{*}}$

$$
\begin{array}{ccc}
\Lambda_{k^{*}}: H^{\frac{1}{2}}(\partial \Omega) & \longrightarrow & H^{-\frac{1}{2}}(\partial \Omega) \\
\varphi & \longrightarrow & k^{*} \partial_{n} u .
\end{array}
$$

Vale ressaltar que poderíamos também estudar um operador de Neumann-to-Dirichlet (NtD), caso fosse dado um fluxo $q^{*}=-k^{*} \partial_{n} u$ sobre $\partial \Omega$ e observado do campo $u^{*}$ sobre $\partial \Omega$.

Na prática é impossível medir os dados sobre toda a fronteira de $\Omega$. Assim, o problema de reconstruir $k^{*}$ deve ser encarado com o conhecimento de uma quantidade limitada de dados.

O problema de EIT é um problema inverso mal-posto por sua natureza física [4]. A complexidade do EIT em comparação com, por exemplo, a tomografia de raios-X tem a propriedade de não-localidade [8], que consiste no fato de que a corrente elétrica não flui no corpo em linha reta, uma alteração local na condutividade elétrica em qualquer lugar no interior do objeto tem um efeito sobre os potenciais de todos os eletrodos de medição.

O objetivo deste trabalho é tratar a reconstrução de $k^{*}$ via análise de sensibilidade topológica. Para isto, supõe-se que existam anomalias em $\Omega$ que tornam a condutividade elétrica constante por partes.

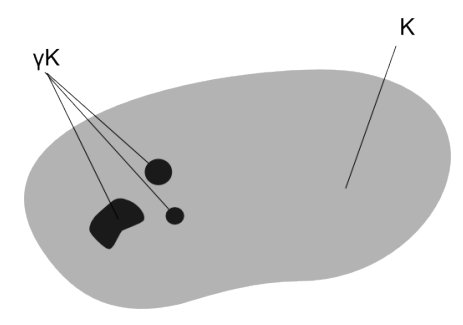

Figura 1: Corpo com anomalias

Assim, o problema de EIT se resume em

Problema 2.1. Encontrar o coeficiente de condutividade elétrica $k^{*}$ tal que

$$
\begin{cases}\operatorname{div}[q(u)]=0 & \text { em } \Omega \\ q(u)=-k^{*} \nabla u, & \\ u=u^{*} & \text { sobre } \partial \Omega \\ q(u) \cdot n=q^{*} & \text { sobre } \partial \Omega\end{cases}
$$

Tem-se assim duas classes de problemas inversos:

- dada uma excitação de Neumann $q^{*}$ sobre $\partial \Omega$, reconstruir $k^{*}$ a partir de observações do campo $u^{*}$ sobre $\partial \Omega$; 
- dada uma excitação de Dirichlet $u^{*}$ sobre $\partial \Omega$, reconstruir $k^{*}$ a partir de observações do fluxo $q^{*}$ sobre $\partial \Omega$.

Será considerada neste trabalho a primeira maneira de tratar o problema. Portanto seja $u$ solução do seguinte problema de Neumann:

$$
\left\{\begin{array}{l}
\operatorname{div}[q(u)]=0 \quad \text { em } \Omega \\
q(u)=-k \nabla u \\
q(u) \cdot n=q^{*} \quad \text { sobre } \partial \Omega \\
\int_{\partial \Omega} q^{*}=0 \\
\int_{\partial \Omega} u=\int_{\partial \Omega} u^{*}
\end{array}\right.
$$

Com forma variacional: Encontrar $u \in U^{N}$ tal que

$$
\int_{\Omega} q(u) \cdot \nabla \eta=\int_{\partial \Omega} q^{*} \cdot \eta, \forall \eta \in V^{N}
$$

onde

$$
U^{N}=\left\{\varphi \in H^{1}(\Omega) ; \int_{\partial \Omega} \varphi=\int_{\partial \Omega} u^{*}\right\} \quad \text { e } \quad V^{N}=\left\{\varphi \in H^{1}(\Omega) ; \int_{\partial \Omega} \varphi=0\right\} .
$$

Consideremos o seguinte funcional de forma:

$$
\psi(\chi)=\mathcal{J}_{\chi}\left(u, u^{*}\right)=\int_{\partial \Omega}\left(u-u^{*}\right)^{2}
$$

onde $u$ é solução de (5), e $\chi$ é a função característica de $\Omega$.

Seja $k^{*}$ o coeficiente de condutividade que se deseja reconstruir.

Proposição 2.1. Seja u solução do problema variacional (5). Então, se $k=k^{*}$, onde $k^{*}$ é uma solução do problema inverso da condutividade (3), tem-se que $u^{*}=u$ sobre $\partial \Omega$ e portanto o funcional $\mathcal{J}_{\chi}$ atinge um minimo.

Tendo em vista a proposição 2.1, observamos que a solução do problema (4) irá coincidir com o dado $u^{*}$ lido na fronteira de $\Omega$ quando $k=k^{*}$, o que implica que o funcional (6) atinge o mínimo. Assim, em encontrar a função $k^{*}$ que minimiza o funcional (6) é equivalente a encontrar o conjunto de inclusões desconhecidas no interior de $\Omega$ considerando $k^{*}$ constante por partes. Uma forma de abordar essa classe de problemas inversos é utilizar o conceito de derivada topológica que será apresentado a seguir.

\section{Análise de Sensibilidade Topológica}

A Análise de Sensibilidade Topológica [6] fornece um desenvolvimento assintótico para um funcional de forma $\psi(\chi)$, cujo termo principal é a derivada topológica, que mede a sensibilidade do funcional com respeito a uma pertubação singular (furo, inclusão, termo-fonte).

\subsection{Derivada Topológica}

Considere um domínio aberto e limitado $\Omega$ sujeito a uma perturbação confinada a uma região $\omega_{\varepsilon}(\hat{x})=\hat{x}+\varepsilon \omega$. Sendo $\hat{x}$ um ponto arbitrário de $\Omega$ e $\omega$ um domínio fixo.

Consideremos a função característica associada ao domínio topologicamente perturbado, $x \mapsto \chi_{\varepsilon}(\hat{x} ; x)$. 


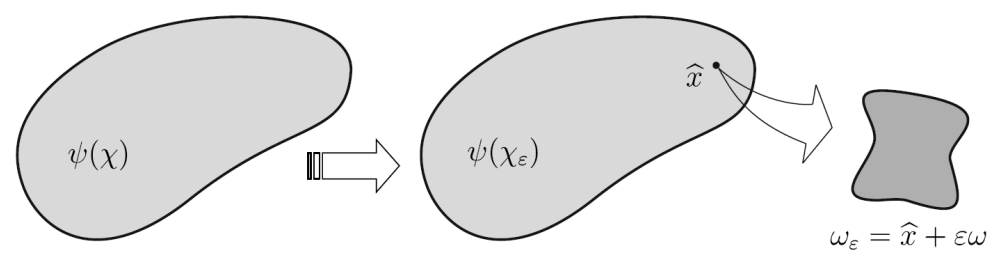

Figura 2: Conceito de derivada topológica

Para o caso do problema inverso de EIT, consideramos inclusões de coeficiente de condutividade constante e têm-se que $\chi_{\varepsilon}(\hat{x})=\mathbb{1}_{\Omega}+(1-\gamma) \mathbb{1}_{\overline{B_{\varepsilon}}(\hat{x})}$, onde $\gamma \in \mathbb{R}^{+}$é o contraste da propriedade material do meio.

Assume-se então, que o funcional de forma associado ao domínio topologicamente perturbado, admite a seguinte expansão assintótica:

$$
\psi\left(\chi_{\varepsilon}(\hat{x})\right)=\psi(\chi)+f_{1}(\varepsilon) \mathcal{T}(\hat{x})+f_{2}(\varepsilon) \mathcal{T}^{2}(\hat{x})+o\left(f_{2}(\varepsilon)\right),
$$

onde

- $\psi(\chi)$ é o funcional de forma associado ao domínio não perturbado;

- $f_{1}(\varepsilon)$ é uma função positiva tal que $f(\varepsilon) \rightarrow 0$ quando $\varepsilon \rightarrow 0$;

- $f_{2}(\varepsilon)$ é uma função positiva de correção de segunda ordem tal que $f_{2}(\varepsilon) \rightarrow 0$ quando $\varepsilon \rightarrow 0$ e $\lim _{\varepsilon \rightarrow 0} \frac{f_{1}(\varepsilon)}{f_{2}(\varepsilon)}=0$

- A função $\hat{x} \mapsto \mathcal{T}(\hat{x})$ é denominada derivada topológica de primeira ordem de $\psi$;

- A função $\hat{x} \mapsto \mathcal{T}^{2}(\hat{x})$ é denominada derivada topológica de segunda ordem de $\psi$;

- O resíduo $o\left(f_{2}(\varepsilon)\right)$ é tal que $\lim _{\varepsilon \rightarrow 0} \frac{o\left(f_{2}(\varepsilon)\right)}{f_{2}(\varepsilon)}=0$;

\subsection{Problema Perturbado}

Considere um domínio perturbado através da nucleação de uma inclusão $B_{\varepsilon}(\hat{x})$ e o funcional de forma associado ao domínio topologicamente perturbado

$$
\psi\left(\chi_{\varepsilon}\right)=\mathcal{J}_{\chi_{\varepsilon}}\left(u_{\varepsilon}, u^{*}\right)=\int_{\partial \Omega}\left(u_{\varepsilon}-u^{*}\right)^{2}
$$

Sendo $u_{\varepsilon}$ é solução de: determinar $u_{\varepsilon} \in U_{\varepsilon}^{N}$ tal que

$$
\int_{\Omega} q_{\varepsilon}\left(u_{\varepsilon}\right) \cdot \nabla \eta=\int_{\partial \Omega} q^{*} \cdot \eta, \forall \eta \in V_{\varepsilon}^{N}
$$

onde

e

$$
U_{\varepsilon}^{N}=\left\{\varphi \in H^{1}(\Omega) ; \int_{\partial \Omega} \varphi=\int_{\partial \Omega} u^{*} \text { e } \llbracket \varphi \rrbracket=0 \text { sobre } \partial B_{\varepsilon}(\hat{x})\right\}
$$

$$
V_{\varepsilon}^{N}=\left\{\varphi \in H^{1}(\Omega) ; \int_{\Omega} \varphi=0 \text { e } \llbracket \varphi \rrbracket=0 \text { sobre } \partial B_{\varepsilon}(\hat{x})\right\} .
$$


A equação de Euler-Lagrange para $u_{\varepsilon}$ é

$$
\begin{cases}\operatorname{div}\left[q_{\varepsilon}\left(u_{\varepsilon}\right)\right]=0 & \text { em } \Omega \\ q_{\varepsilon}\left(u_{\varepsilon}\right)=-\gamma_{\varepsilon} k \nabla u_{\varepsilon} & \\ q_{\varepsilon}\left(u_{\varepsilon}\right) \cdot n=q^{*} & \text { sobre } \partial \Omega \\ \int_{\partial \Omega} q^{*}=0 & \\ \int_{\partial \Omega} u_{\varepsilon}=\int_{\partial \Omega} u^{*} & \\ \llbracket u_{\varepsilon} \rrbracket=0 & \text { sobre } \partial B_{\varepsilon} \\ \llbracket q_{\varepsilon}\left(u_{\varepsilon}\right) \rrbracket \cdot n=0 & \text { sobre } \partial B_{\varepsilon},\end{cases}
$$

sendo

$$
\gamma_{\varepsilon}= \begin{cases}1 & \text { se } x \in \Omega \backslash \overline{B_{\varepsilon}(\hat{x})} \\ \gamma & \text { se } x \in B_{\varepsilon}(\hat{x})\end{cases}
$$

e $\gamma$ um parâmetro real positivo.

\section{Resultados Obtidos}

Afim de identificar $f_{1}(\varepsilon), f_{2}(\varepsilon)$, calcular $\mathcal{T}(\hat{x})$ e $\mathcal{T}^{2}(\hat{x})$ em $(7)$ necessário conhecer o comportamento de $u_{\varepsilon}$ com respeito ao parâmetro $\varepsilon$.

\subsection{Expansão Assintótica}

Apresenta-se neste momento a expansão assintótica de $u_{\varepsilon}$. Para uma justificativa mais rigorosa das expansões assintóticas apresentadas, ver [3] e [5].

Para expandirmos $u_{\varepsilon}$, postula-se um ansatz, e em seguida verifica-se que tal expansão é suficiente para se determinar as derivadas topológicas de primeira e segunda ordem.

Assim sendo, consideremos o seguinte ansatz:

$$
u_{\varepsilon}(x)=u(x)+w_{\varepsilon}(x / \varepsilon)+\varepsilon^{2} \tilde{u}(x)+\tilde{w}_{\varepsilon}(x / \varepsilon)+\varepsilon^{4} \tilde{\tilde{u}}(x)+\tilde{\tilde{u}}_{\varepsilon}(x) .
$$

Expandindo em Taylor $u(x)$ construímos os problemas associados aos termos da expansão (12), ou seja, $w_{\varepsilon}(x / \varepsilon), \tilde{u}(x), \tilde{w}_{\varepsilon}(x / \varepsilon), \tilde{\tilde{u}}(x)$ e $\tilde{\tilde{u}}_{\varepsilon}(x)$ que são, respectivamente, soluções dos seguintes problemas

$$
\begin{cases}\operatorname{div}\left[q_{\varepsilon}\left(w_{\varepsilon}\right)\right]=0 & \text { no } \mathbb{R}^{2} \\ q_{\varepsilon}\left(w_{\varepsilon}\right)=-\gamma_{\varepsilon} k \nabla w_{\varepsilon} & \text { no } \mathbb{R}^{2} \\ w_{\varepsilon} \rightarrow 0 & \text { no } \infty \\ \llbracket w_{\varepsilon} \rrbracket=0 & \text { sobre } \partial B_{\varepsilon}(\hat{x}) \\ \llbracket q_{\varepsilon}\left(w_{\varepsilon}\right) \rrbracket \cdot n=d & \text { sobre } \partial B_{\varepsilon}(\hat{x}),\end{cases}
$$

onde $d=k(1-\gamma) \nabla u(\hat{x}) \cdot n$.

$$
\begin{cases}\operatorname{div}[q(\tilde{u})]=0, & \text { em } \Omega, \\ q(\tilde{u})=-k \nabla \tilde{u}, & \text { em } \Omega, \\ \int_{\partial \Omega} \tilde{u}=-\int_{\partial \Omega} g & \\ q(\tilde{u}) \cdot n=-q(g) \cdot n, & \text { sobre } \partial \Omega\end{cases}
$$

onde $g=\frac{1-\gamma}{1+\gamma} \frac{1}{\|x-\hat{x}\|^{2}} \nabla u(\hat{x}) \cdot(x-\hat{x})$.

$$
\begin{cases}\operatorname{div}\left[q_{\varepsilon}\left(\tilde{w}_{\varepsilon}\right)\right]=0, & \text { no } \mathbb{R}^{2} \\ q_{\varepsilon}\left(\tilde{w}_{\varepsilon}\right)=-\gamma_{\varepsilon} k \nabla \tilde{w}_{\varepsilon} & \text { no } \mathbb{R}^{2} \\ \tilde{w}_{\varepsilon} \rightarrow 0, & \text { no } \infty \\ \llbracket \tilde{w}_{\varepsilon} \rrbracket=0, & \text { sobre } \partial B_{\varepsilon} \\ \llbracket q_{\varepsilon}\left(\tilde{w}_{\varepsilon}\right) \rrbracket \cdot n=e & \text { sobre } \partial B_{\varepsilon},\end{cases}
$$

onde $e=-\varepsilon(1-\gamma) k \nabla^{2} u(\hat{x}) n \cdot n$.

$$
\begin{cases}\operatorname{div}\left[q_{\varepsilon}\left(\tilde{\tilde{u}}_{\varepsilon}\right)\right]=0 & \text { em } \Omega \\ q_{\varepsilon}\left(\tilde{\tilde{u}}_{\varepsilon}\right)=-\gamma_{\varepsilon} k \nabla \tilde{\tilde{u}}_{\varepsilon} & \text { em } \Omega \\ q_{\varepsilon}\left(\tilde{\tilde{u}}_{\varepsilon}\right) \cdot n=0 & \text { sobre } \partial \Omega \\ \int_{\partial \Omega} \tilde{\tilde{u}}_{\varepsilon}=0 & \\ \llbracket \tilde{u}_{\varepsilon} \rrbracket=0 & \text { sobre } \partial B_{\varepsilon} \\ \llbracket q_{\varepsilon}\left(\tilde{\tilde{u}}_{\varepsilon}\right) \rrbracket \cdot n=f & \text { sobre } \partial B_{\varepsilon},\end{cases}
$$

onde $f=\varepsilon^{2}(1-\gamma) k \nabla^{3} u(\xi) n^{3}$.

$$
\begin{cases}\operatorname{div}[q(\tilde{\tilde{u}})]=0 & \text { em } \Omega, \\ q(\tilde{\tilde{u}})=-k \nabla \tilde{\tilde{u}} & \text { em } \Omega, \\ q(\tilde{\tilde{u}}) \cdot n=-q(h) \cdot n, & \text { sobre } \partial \Omega \\ \int_{\partial \Omega} \tilde{\tilde{u}}=-\int_{\partial \Omega} h & ,\end{cases}
$$


Os problemas (13) e (15) possuem soluções explícitas e a partir destas constrói-se os problemas (14) e (17) que estão associados ao domínio não perturbado que são resolvidos computacionalmente. Já a solução $\tilde{\tilde{u}}_{\varepsilon}$ do problema (16) não contribui para o cálculo das derivadas topológicas de primeira e segunda ordem, gerando assim um resíduo na expansão.

Considerando (12) obtêm-se a seguinte expansão para o funcional associado ao domínio perturbado.

$$
\begin{aligned}
\psi\left(\chi_{\varepsilon}\right)=\psi(\chi)+2 \varepsilon^{2} \int_{\partial \Omega}\left(u-u^{*}\right)(g+\tilde{u})+2 \varepsilon^{4} \int_{\partial \Omega}\left(u-u^{*}\right)(h+ & +\tilde{\tilde{u}}) \\
& +\varepsilon^{4} \int_{\partial \Omega}(g+\tilde{u})^{2}+o\left(\varepsilon^{4}\right) .
\end{aligned}
$$

Note que os problemas associados a $\tilde{u}$ e a $\tilde{\tilde{u}}$ que aparecem na primeira e segunda integral acima deveriam ser resolvidos para cada ponto $\hat{x}$. Para evitar este custo, de resolver uma EDP para cada ponto, pode-se substituir esses termos por estados adjuntos que não dependem do ponto. Todavia, como a terceira integral envolve um termo quadrático em $\tilde{u}$ não podemos substitui-lo por um estado adjunto. Portanto substituindo apenas o termo que envolve $\tilde{\tilde{u}}$, por um estado adjunto $v$ solução de

$$
\begin{cases}\operatorname{div}[q(v)]=0 & \text { em } \Omega \\ q(v)=-k \nabla v & \\ q(v) \cdot n=2\left(u-u^{*}\right) & \text { sobre } \partial \Omega \\ \int_{\partial \Omega} v=0 & \end{cases}
$$

É fácil ver, variacionalmente, que $v$ satisfaz

$$
2 \int_{\partial \Omega}\left(u-u^{*}\right) \tilde{\tilde{u}}=-\int_{\partial \Omega} q(h) \cdot n v
$$

de forma que podemos reescrever (18) como

$$
\psi\left(\chi_{\varepsilon}\right)=\psi(\chi)+\underbrace{\varepsilon^{2}}_{f_{1}(\varepsilon)} \underbrace{2 \int_{\partial \Omega}\left(u-u^{*}\right)(g+\tilde{u})}_{\mathcal{T}(\hat{x})}+\underbrace{\varepsilon^{4}}_{f_{2}(\varepsilon)}[\underbrace{2 \int_{\partial \Omega}\left(u-u^{*}\right) h-\int_{\partial \Omega} q(h) \cdot n v+\int_{\partial \Omega}(g+\tilde{u})^{2}}_{\mathcal{T}^{2}(\hat{x})}]
$$

Na figura 3, apresenta-se a solução exata do problema inverso, a derivada topológica de primeira ordem e a expansão (20) sem o resíduo.

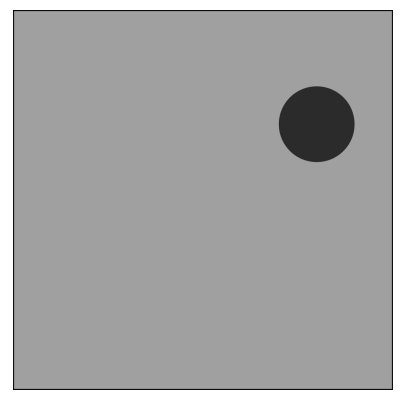

(a) Solução exata

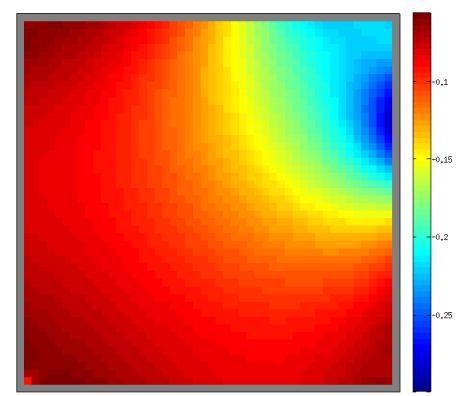

(b) Derivada Topológica

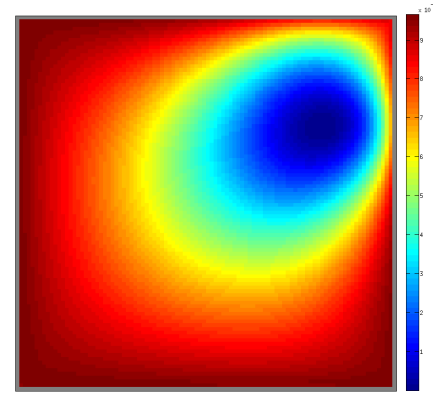

(c) Expansão (20) sem o resíduo

Figura 3: Solução exata a ser encontrada, Derivada Topológica de Primeira Ordem e expansão (20) 
Observa-se que derivada topológica de primeira ordem não é suficiente para detectarmos a anomalia, uma vez que ela atinge os valores mais críticos na fronteira (Figura 3b). Considerando a derivada topológica de segunda ordem que consiste de termos não locais, as referidas discrepâncias são corrigidas (3c), de forma que funcional associado ao problema perturbado atinge seu mínimo exatamente no centro da anomalia.

Como os dados na fronteira são conhecidos, o cálculo das derivadas é feito apenas para os elementos do interior do domínio conforme as figuras 3b e 3c.

\section{Conclusão}

Neste trabalho apresentou-se a determinação das derivadas topológicas de primeira e segunda ordem para um funcional de forma que mede a diferença entre os potenciais medidos e calculados numericamente de uma equação diferencial parcial. Esta EDP representa o comportamento de um campo elétrico em um domínio $\Omega$ quando este é submetido a um conjunto de fluxo elétrico. A escolha do funcional de forma adotado deve-se ao fato de que dispomos de informações restritas a fronteira do domínio. Viu-se que a minimização deste funcional conduz a solução do problema inverso de tomografia por impedância elétrica.

Uma forma de tratar esse problema de minimização é utilizar o conceito de derivada topológica, o qual foi apresentado na seção 3. A derivada topológica de primeira ordem é utilizada como direção de descida na minimização do funcional de forma. Porém, esta derivada não é suficiente para reconstruir a solução do problema inverso, uma vez que seus valores mais negativos são atingidos na fronteira do domínio.

Visto isso, introduziu-se mais um termo na expansão do funcional de forma associado ao domínio não perturbado, a saber a derivada topológica de segunda ordem que corrige as discrepâncias na fronteira do domínio apresentadas pela derivada topológica de primeira ordem.

\section{Referências}

[1] M. Bertero and P. Boccacci. Introduction to inverse problems in imaging. Institute of Physics Publishing, Bristol, 1998.

[2] V. Isakov. Inverse problems for partial differential equations, second ed. Applied Mathematical Sciences, Springer, New York, 2006.

[3] V. A. Kozlov, V. G. Maz'ya, and A. B. Movchan. Asymptotic analysis of fields in multistructures. Clarendon Press, Oxford, 1999.

[4] Vauhkonen M. Electrical impedance tomography and prior information. Ph.D. Thesis, Kuopio University, Finland, 1997.

[5] W. G. Mazja, S. A. Nasarow, and B. A. Plamenewski. Asymptotics of solutions to elliptic boundary-value problems under a singular perturbation of the domain. Tbilisi University, Tbilisi, 1981. (in Russian).

[6] A. A. Novotny and J. Sokołowski. Topological derivatives in shape optimization. Interaction of Mechanics and Mathematics. Springer, 2013.

[7] H. Grossauer M. Haltmeier O. Scherzer, M. Grasmair and F. Lenzen. Variational methods in imaging. Applied Mathematical Sciences, Springer, New York, 2009.

[8] N. Polydorides. Image Reconstruction Algorithms for Soft-Field Tomography. Ph.D. Thesis, Manchester, United Kingdom, 2002. 